\title{
Variability of $\alpha / \beta$ Ratios for Prostate Cancer With the Fractionation Schedule: Caution Against Using the Linear-Quadratic Model for Hypofractionated Radiotherapy
}

\author{
Ming Cui \\ Department of Radiation Oncology Gastrointestinal and Urinary and Musculoskeletal Cancer, Cancer Hospital of China Medical \\ University, Shenyang, Liaoning, P.R. China \\ Xianshu Gao ( $\square$ doctorgaoxs@126.com ) \\ Peking University First Hospital https://orcid.org/0000-0002-8348-456X \\ Xiaoying Li \\ Department of Radiation Oncology, Peking University First Hospital Peking University, Beijing China \\ Mingwei Ma \\ Department of Radiation Oncology, Peking University First Hospital, Peking University, Beijing, China \\ Xin Qi \\ Department of Radiation Oncology, Peking University First Hospital, Peking University, Beijing, China. \\ Yuta Shibamoto \\ Department of Radiology, Nagoya City University Graduate School of Medical Sciences, Nagoya 467-8601, Japan.
}

\section{Research}

Keywords: LQ model, $\alpha / \beta$ ratio, Hypofractionated radiotherapy, High dose per fraction, Prostate cancer

Posted Date: August 3rd, 2021

DOI: https://doi.org/10.21203/rs.3.rs-750755/v1

License: @ (i) This work is licensed under a Creative Commons Attribution 4.0 International License. Read Full License

Version of Record: A version of this preprint was published at Radiation Oncology on March 18th, 2022. See the published version at https://doi.org/10.1186/s13014-022-02010-9. 


\section{Abstract}

Background: Prostate cancer ( $\mathrm{PCa}$ ) is known to be suitable for hypofractionated radiotherapy due to the very low a/ $\beta$ ratio (about 1.5-3 Gy). However, several randomized controlled trials have not shown the superiority of hypofractionated radiotherapy over conventionally fractionated radiotherapy. Besides, in vivo and in vitro experimental results show that the linear-quadratic (LQ) model may not be appropriate for hypofractionated radiotherapy, and we guess it may be due to the influence of fractionation schedules on the $\alpha / \beta$ ratio. Therefore, this study attempted to estimate the $\alpha / \beta$ ratio in different fractionation schedules and evaluate the applicability of the $L Q$ model in hypofractionated radiotherapy.

Methods: The maximum likelihood principle in mathematical statistics was used to fit the parameters: $k$, $a$ and $\beta$ values in the tumor control probability (TCP) formula derived from the LQ model. In addition, the fitting results were substituted into the original TCP formula to calculate 5-year biochemical relapse-free survival for further verification.

Results: Information necessary for fitting could be extracted from a total of 23,281 PCa patients. A total of 16,442 PCa patients were grouped according to fractionation schedules. We found that, for patients who received conventionally fractionated radiotherapy, moderately hypofractionated radiotherapy, and stereotactic body radiotherapy, the average $\alpha / \beta$ ratios were 1.78 Gy $(95 \% \mathrm{Cl}: 1.59-1.98, P$ < 0.001), 3.46 Gy (95\% Cl: 3.08-3.83, $P<0.001)$, and $4.24 \mathrm{~Gy}$ (95\% Cl: $4.10-4.39, P<0.001)$, respectively. Hence, the calculated a/ $\beta$ ratios for PCa tended to become higher when the dose per fraction increased. Among all PCa patients, 14,641 could be grouped according to the risks of $\mathrm{PCa}$ in patients receiving radiotherapy with different fractionation schedules. The results showed that as the risk increased, the $k$ and $a$ values decreased, indicating that the number of effective target cells decreased and the radioresistance increased.

Conclusions: The LQ model appeared to be inappropriate for high doses per fraction owing to $\alpha / \beta$ ratios tending to become higher when the dose per fraction increased. Therefore, to convert the conventionally fractionated radiation doses to equivalent high doses per fraction using the standard LQ model, a higher $\alpha / \beta$ ratio should be used for calculation.

\section{Background}

With the development of high-precision radiotherapy, fractionation schedules to treat various tumors are changing [1]. Hypofractionated radiotherapy is being increasingly employed in clinics as stereotactic body radiotherapy (SBRT) and moderately hypofractionated intensity-modulated radiotherapy (IMRT), which have become valuable therapeutic approaches for a variety of tumors owing to the improved dose distribution. In addition, for prostate cancer (PCa), hypofractionated IMRT and SBRT seem to have radiobiological advantages based on the linear-quadratic (LQ) model estimation.

Since definitive hypofractionated radiotherapy is a relatively novel treatment, optimal dose fractionation schedules often need to be inferred from mathematical calculation, and an LQ model-based formula is frequently used to convert the conventionally fractionated radiation doses to high doses per fraction by clinicians due to its convenience and simplicity [2]. Recently, however, several investigators demonstrated that the standard LQ model may not be applicable to hypofractionated radiotherapy especially in SBRT [2-6], although other researchers insist that the LQ model can be used to estimate the antitumor effects of hypofractionated radiotherapy $[7,8]$.

The $\alpha / \beta$ ratio is a key factor in the LQ model [2]. Basically, the $\alpha / \beta$ ratio of a tumor is obtained from an in vitro dose-survival curve of tumor cells $[2,6]$, but this method cannot be applied to human tumors in patients. The $\alpha / \beta$ ratio can also be obtained from in vivo tumor or normal tissue responses to different fractionation schedules, and following this in vivo method, a mathematical method was elaborated to estimate the $\alpha / \beta$ ratio from clinical data employing various fractionation schedules $[9,10]$. Using the method, the $\alpha / \beta$ ratios for various tumors have been reported, and PCa was found to have a low $\alpha / \beta$ ratio $[9,10]$, which was lower than the $\alpha / \beta$ ratio for normal tissue late reactions. Accordingly, moderately hypofractionated IMRT and SBRT are being increasingly used in the treatment of PCa. However, since the reliability of the LQ model in SBRT was questioned in recent studies [4-6], it may be necessary to re-evaluate the validity of converting conventionally fractionated doses to hypofractionated doses with the LQ model. Previous studies only tried to demonstrate that $\mathrm{PCa}$ has a low $\alpha / \beta$ ratio [9-12], and variability of the $\alpha / \beta$ ratios with the dose per fraction has not been investigated. Therefore, we carried out an analysis using an established mathematical calculation method to estimate the variation in the $\alpha / \beta$ ratio of $\mathrm{PCa}$ according to the daily fractional dose.

\section{Methods}

\section{Clinical data collection}

Page 2/15 
We searched for relevant articles in PubMed with key words of "radiotherapy" or "radiation therapy" and "prostate cancer" or "prostatic carcinoma". The inclusion criteria were as follows: (1) patients with PCa undergoing conventionally fractionated radiotherapy, moderately hypofractionated radiotherapy, or SBRT, with or without androgen deprivation therapy (ADT) and (2) 5-year biochemical relapse-free survival (5y-bRFS), number of patients, total dose, and fraction number or dose per fraction available from the articles. Articles that lacked the necessary fitting data or that used other fractionation schedules, such as hyperfractionated radiotherapy, were excluded. Conventionally fractionated radiotherapy was defined as that using 1.8-2.1 Gy per fraction. Moderately hypofractionated radiotherapy was defined as that using 2.19-3.5 Gy in our study. SBRT was defined as that using 6.5 Gy per fraction or greater according to National Comprehensive Cancer Network (NCCN) guidelines. Five-year bRFS according to the ASTRO or Phoenix definition was evaluated. The ASTRO definition of biochemical relapse is three consecutive rises in prostate-specific antigen (PSA) from the nadir [13]. The Phoenix definition of biochemical relapse is a rise of PSA over $2 \mathrm{ng} / \mathrm{mL}$ from the nadir [14]. Risk stratification of PCa was mostly made according to the NCCN guidelines risk group classification and in part D'Amico's classification.

\section{Estimation of the $\alpha / \beta$ ratios}

Statistical analyses were carried out exactly following the method of Miralbell and coworkers $[15,16]$. Briefly, standard LQ models for tumor control at 5 years of the form:

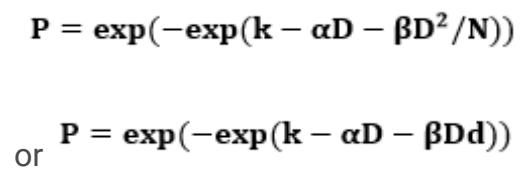

were fitted to the obtained data. In the formula, $\mathrm{P}$ is interpreted as the tumor control probability (5y-bRFS); $\mathrm{D}$ is the total dose; $\mathrm{N}$ is the number of fractions during the whole radiotherapy; $d$ is the dose per fraction; and $k$ represents the natural logarithm of an effective target cell number. a represents unrepairable lethal damage caused by a one-track action and $\beta$ represents repairable sublethal damage caused by a two-track action in the DNA damage repair kinetics [17]. So, the $\alpha / \beta$ ratio can be considered as the balance between the two forms of damage.

Fitting was performed by maximum-likelihood methods and parameter estimates were obtained using the custom-written code in Stata version $12.0[15,16,18]$. The parameter estimation was directed by Professor Geng and Dr. Yin of the School of Mathematical Sciences, Peking University.

In order to reduce the errors of fitting parameters, we removed one group every time from the 22 groups of data (taking conventionally fractionated radiotherapy group as an example); we could get 22 groups of sample data, which is C21 22in mathematics. Using these 22 groups of data, the average and standard error of the sample can be obtained, and then the population mean and the confidence interval of fitting parameters can be obtained. The advantage of this method is that the confidence interval of the fitting parameters can be obtained and the fitting error can be reduced by estimating the population mean from the sample mean. For the parameters of the LQ model, significant figures were kept to be 3 , or were rounded to the 2 nd decimal place.

In order to verify the accuracy of the results, we substituted the fitting results: $k, a$ and $\alpha / \beta$ values, and known parameters, i.e., total dose (D), single dose (d) and total fraction number (N), into the original TCP formula (1) or (2) and got a calculated P (5y-bRFS). Using goodness of fit test by chi-square test, it was checked whether there was statistical difference between the calculated $\mathrm{P}$ and the $\mathrm{P}$ from the original study. The statistical software SPSS 22.0 was used.

\section{Results}

There were 45 articles(23,281 PCa patients)incorporated in this study published during 2003 to February 2021. Detailed information on the 45 articles is shown in Table 1. Among them, 5 articles reported on more than 1,000 patients [19-23], and 3 reported on 500-1,000 patients [24-26]. Among all 45 studies, a total of 38 articles (16,442 PCa patients) could be grouped according to fractionation schedules of radiotherapy and complete information could be extracted based on the inclusion and exclusion criteria; 15 were enrolled in the conventionally fractionated radiotherapy group, 24 were enrolled in the moderately hypofractionated radiotherapy group, and 8 were enrolled in the SBRT group. Nine articles were duplicated since the studies investigated both conventional fractionation and moderate hypofractionation. Detailed data from each article are shown in Table 2-4 according to the three different regimens of radiotherapy. To explore the relationship between $\alpha / \beta$ ratios and risks of $\mathrm{PCa}$, we also divided each group into three subgroups by the 
risks in patients receiving radiotherapy with different fractionation schedules. Of all the 45 studies, 21 studies (14,641 PCa patients) could be grouped and the characteristics are shown in Supplementary Tables 1 to 3. 
Table 1

Detailed information on 45 cited articles

\begin{tabular}{|c|c|c|c|c|c|}
\hline Study & First author & Year & Journal $^{a}$ & Volume & Pages \\
\hline 1 & Aizer & 2009 & Radiother Oncol & 93 & $185-191$ \\
\hline 2 & Alicikus & 2011 & Cancer & 117 & $1429-1437$ \\
\hline 3 & Cahlon & 2008 & IJROBP & 71 & $330-337$ \\
\hline 4 & Eade & 2007 & IJROBP & 68 & $682-689$ \\
\hline 5 & Kuban & 2008 & IJROBP & 70 & $67-74$ \\
\hline 6 & Lukka & 2005 & $\mathrm{~J}$ Clin Oncol & 23 & $6132-6138$ \\
\hline 7 & Valdagni & 2005 & Radiother Oncol & 75 & $74-82$ \\
\hline 8 & Zelefsky & 2008 & IJROBP & 71 & $1028-1033$ \\
\hline 9 & Miralbell & 2012 & IJROBP & 82 & e17-24 \\
\hline 10 & Arcangeli & 2012 & IJROBP & 84 & $1172-1178$ \\
\hline 11 & Catton & 2017 & J Clin Oncol & 35 & $1884-1890$ \\
\hline 12 & Dearnaley & 2016 & The Lancet Oncology & 17 & $1047-1060$ \\
\hline 13 & Incrocci & 2016 & The Lancet Oncology & 17 & $1061-1069$ \\
\hline 14 & Kim & 2014 & Radiat Oncol J & 32 & $187-197$ \\
\hline 15 & Kupelian & 2005 & IJROBP & 63 & $1463-1468$ \\
\hline 16 & Leborgne & 2009 & IJROBP & 74 & $1441-1446$ \\
\hline 17 & Leborgne & 2012 & IJROBP & 82 & $1200-1207$ \\
\hline 18 & Pollack & 2013 & J Clin Oncol & 31 & $3860-3868$ \\
\hline 19 & Yeoh & 2006 & IJROBP & 66 & $1072-1083$ \\
\hline 20 & Cheung & 2016 & IJROBP & 96 & S33 \\
\hline 21 & Di Muzio & 2016 & Clin Oncol (R Coll Radiol) & 28 & $490-500$ \\
\hline 22 & Faria & 2011 & Radiother Oncol & 101 & $486-489$ \\
\hline 23 & Fonteyne & 2012 & IJROBP & 84 & e483-490 \\
\hline 24 & Hashimoto & 2017 & Int J Clin Oncol & NA & NA \\
\hline 25 & Kuban & 2010 & IJROBP & 78 & S58-59 \\
\hline 26 & Kupelian & 2007 & IJROBP & 68 & $1424-1430$ \\
\hline 27 & Lieng & 2017 & Radiother Oncol & 122 & $93-98$ \\
\hline 28 & Livsey & 2003 & IJROBP & 57 & $1254-1259$ \\
\hline 29 & Mai & 2010 & IJROBP & 78 & S59 \\
\hline 30 & Patel & 2013 & IJROBP & 86 & $534-539$ \\
\hline 31 & Pervez & 2017 & Am J Clin Oncol & 40 & $200-206$ \\
\hline 32 & Shimizu & 2017 & Anticancer Res & 37 & $5829-5835$ \\
\hline 33 & Thomson & 2012 & Prostate Cancer & NA & NA \\
\hline 34 & Viani & 2016 & Rep Pract Oncol Radiother & 21 & $162-167$ \\
\hline
\end{tabular}

a: Journal abbreviations follow the PubMed style. IJROBP = Int J Radiat Oncol Biol Phys. NA: not available. 


\begin{tabular}{|lllllll|}
\hline Study & First author & Year & Journal $^{a}$ & Volume & Pages \\
\hline 35 & Bolzicco & 2013 & BMC Urol & 13 & NA \\
\hline 36 & Fuller & 2014 & Front Oncol & 4 & NA \\
\hline 37 & Kang & 2011 & Tumori & 97 & $43-48$ \\
\hline 38 & Katz & 2013 & Radiat Oncol & 8 & NA \\
\hline 39 & King & 2013 & Radiother Oncol & 109 & $217-221$ \\
\hline 40 & Lee & 2014 & Medicine (Baltimore) & 93 & e290 \\
\hline 41 & Loblaw & 2013 & Radiother Oncol & 107 & $153-158$ \\
\hline 42 & Mantz & 2014 & Front Oncol & 96 & NA \\
\hline 43 & Meier & 2016 & IJROBP & 158 & $184-190$ \\
\hline 44 & Tsang & 2021 & Radiother Oncol & 107 & $288-296$ \\
\hline 45 & Chin,S & 2020 & IJROBP & 45 \\
\hline a: Journal abbreviations follow the PubMed style. IJROBP = Int J Radiat Oncol Biol Phys. NA: not available. \\
\hline
\end{tabular}


Table 2

Conventionally fractionated radiotherapy group

\begin{tabular}{|c|c|c|c|c|c|c|c|}
\hline Study number & Author & Number of patients & $5 y$-bRFS & Total dose (D) & $\begin{array}{l}\text { Fractions }(\mathrm{N}) / \\
\text { single dose }\end{array}$ & $\begin{array}{l}D^{2} / N \\
\text { (C) }\end{array}$ & Definition of bRFS ${ }^{b}$ \\
\hline 1 & Aizer & 352 & 0.748 & 75.6 & 42 & 136.08 & $P$ \\
\hline 2 & Arcangeli & 85 & 0.79 & 80 & 40 & 160 & $P$ \\
\hline 3 & Catton & 598 & 0.85 & 78 & 39 & 156 & $P$ \\
\hline 4 & Dearnaley & 1065 & 0.883 & 74 & 37 & 148 & $\mathrm{P}$ \\
\hline \multirow[t]{4}{*}{5} & Eade & 43 & 0.7 & 69 & 2.1 & 144.9 & $\mathrm{P}$ \\
\hline & Eade & 552 & 0.81 & 72.5 & 2.1 & 152.25 & $\mathrm{P}$ \\
\hline & Eade & 568 & 0.83 & 77.5 & 2.1 & 162.75 & $\mathrm{P}$ \\
\hline & Eade & 367 & 0.89 & 81 & 2.1 & 170.1 & $\mathrm{P}$ \\
\hline 6 & Incrocci & 397 & 0.771 & 78 & 39 & 156 & $P$ \\
\hline 7 & Kim & 56 & 0.641 & 70.2 & 39 & 126.36 & $\mathrm{P}$ \\
\hline \multirow[t]{2}{*}{8} & Kuban & 150 & 0.78 & 70 & 35 & 140 & $\mathrm{P}$ \\
\hline & Kuban & 151 & 0.85 & 78 & 39 & 156 & $P$ \\
\hline 9 & Kupelian & 310 & 0.78 & 78 & 39 & 156 & $A$ \\
\hline 10 & Leborgne & 160 & 0.887 & 78 & 39 & 156 & $\mathrm{P}$ \\
\hline 11 & Lukka & 470 & 0.4705 & 66 & 33 & 132 & A \\
\hline 12 & Pollack & 152 & 0.852 & 76 & 38 & 152 & $P$ \\
\hline 13 & Valdagni & 161 & 0.7 & 74 & 37 & 148 & $A$ \\
\hline 14 & Yeoh & 109 & 0.555 & 64 & 32 & 128 & $A$ \\
\hline \multirow[t]{4}{*}{15} & Zelefsky & 358 & 0.61 & 70.2 & 39 & 126.36 & $P$ \\
\hline & Zelefsky & 471 & 0.74 & 75.6 & 42 & 136.08 & $P$ \\
\hline & Zelefsky & 741 & 0.85 & 81 & 45 & 145.8 & $P$ \\
\hline & Zelefsky & 477 & 0.82 & 86.4 & 48 & 155.52 & $P$ \\
\hline
\end{tabular}


Table 3

Moderately hypofractionated radiotherapy group

\begin{tabular}{|c|c|c|c|c|c|c|c|}
\hline Study number & Author & Number of patients & $5 y-b R F S$ & Total dose (D) & $\begin{array}{l}\text { Fractions } \\
\text { (N) }\end{array}$ & $D^{2} / N(C)$ & Definition of $\mathrm{bRFS}^{b}$ \\
\hline 1 & Arcangeli & 83 & 0.85 & 62 & 20 & 192.2 & $P$ \\
\hline 2 & Catton & 608 & 0.85 & 60 & 20 & 180 & $P$ \\
\hline 3 & Cheung & 230 & 0.837 & 67.5 & 25 & 182.25 & $P$ \\
\hline 4 & Chin,S & 112 & 0.68 & 52.5 & 20 & 137.8125 & $P$ \\
\hline \multirow[t]{2}{*}{5} & Dearnaley & 1077 & 0.859 & 57 & 19 & 171 & $P$ \\
\hline & Dearnaley & 1074 & 0.906 & 60 & 20 & 180 & $\mathrm{P}$ \\
\hline \multirow[t]{3}{*}{6} & Di Muzio & 80 & 0.911 & 74.2 & 28 & 196.63 & $P$ \\
\hline & Di Muzio & 78 & 0.946 & 71.4 & 28 & 182.07 & $P$ \\
\hline & Di Muzio & 53 & 0.962 & 74.2 & 28 & 196.63 & $P$ \\
\hline 7 & Faria & 82 & 0.954 & 66 & 22 & 198 & $P$ \\
\hline 8 & Fonteyne & 113 & 0.94 & 56 & 16 & 196 & $P$ \\
\hline 9 & Hashimoto & 195 & 0.924 & 66 & 22 & 198 & $\mathrm{P}$ \\
\hline \multirow[t]{2}{*}{10} & Lieng & 96 & 0.81 & 60 & 20 & 180 & $\mathrm{P}$ \\
\hline & Lieng & 27 & 0.88 & 66 & 22 & 198 & $P$ \\
\hline 11 & Incrocci & 407 & 0.805 & 64.6 & 19 & 219.64 & $P$ \\
\hline 12 & Kim & 30 & 0.929 & 70 & 28 & 175 & $P$ \\
\hline 13 & Kuban & 102 & 0.96 & 72 & 30 & 172.8 & A \\
\hline 14 & Kupelian & 100 & 0.88 & 70 & 28 & 175 & $P$ \\
\hline 15 & Kupelian & 770 & 0.83 & 70 & 28 & 175 & $\mathrm{P}$ \\
\hline 16 & Leborgne & 114 & 0.894 & 61.2 & 20 & 187.272 & $P$ \\
\hline 17 & Mai & 596 & 0.927 & 76.65 & 35 & 167.8635 & $P$ \\
\hline 18 & Patel & 129 & 0.97 & 66 & 22 & 198 & $P$ \\
\hline 19 & Pervez & 60 & 0.9167 & 67.5 & 25 & 182.25 & $\mathrm{P}$ \\
\hline 20 & Pollack & 151 & 0.81 & 70.2 & 26 & 189.54 & $P$ \\
\hline \multirow[t]{3}{*}{21} & Shimizu & 73 & 0.77 & 74.8 & 34 & 164.56 & $P$ \\
\hline & Shimizu & 21 & 0.92 & 74.8 & 34 & 164.56 & $P$ \\
\hline & Shimizu & 44 & 0.95 & 72.6 & 33 & 159.72 & $P$ \\
\hline \multirow[t]{2}{*}{22} & Thomson & 30 & 0.5 & 57 & 19 & 171 & $P$ \\
\hline & Thomson & 30 & 0.58 & 60 & 20 & 180 & $P$ \\
\hline 23 & Viani & 149 & 0.946 & 69 & 23 & 207 & $P$ \\
\hline 24 & Yeoh & 108 & 0.574 & 55 & 20 & 151.25 & A \\
\hline
\end{tabular}


SBRT group

\begin{tabular}{|c|c|c|c|c|c|c|c|}
\hline $\begin{array}{l}\text { Study } \\
\text { number }\end{array}$ & Author & Number of patients & $5 y-b R F S$ & Total dose (D) & Fractions $(\mathrm{N})$ & $D^{2} / N(C)$ & Definition of bRFS ${ }^{b}$ \\
\hline 1 & Bolzicco & 100 & 0.944 & 35 & 5 & 245 & $P$ \\
\hline 2 & Kang & 44 & 0.936 & 34 & 4 & 289 & $P$ \\
\hline \multirow[t]{4}{*}{3} & King & 1100 & 0.93 & 36.25 & 5 & 262.8 & $P$ \\
\hline & King & 385 & 0.925 & 35 & 5 & 245 & $P$ \\
\hline & King & 589 & 0.907 & 36.25 & 5 & 262.8 & $P$ \\
\hline & King & 126 & 0.958 & 39 & 5 & 304.2 & $P$ \\
\hline 4 & Lee & 45 & 0.897 & 36 & 5 & 259.2 & $P$ \\
\hline 5 & Loblaw & 84 & 0.98 & 35 & 5 & 245 & $P$ \\
\hline 6 & Mantz & 102 & 1 & 40 & 5 & 320 & $P$ \\
\hline 7 & Meier & 309 & 0.971 & 40 & 5 & 320 & $P$ \\
\hline 8 & Tsang & 43 & 0.92 & 36.25 & 5 & 262.8 & $P$ \\
\hline
\end{tabular}

Estimated $\alpha / \beta$ ratios are shown in Table 5. Among all 16,442 PCa patients, 7,793 patients received conventionally fractionated radiotherapy, and the average $\alpha / \beta$ ratio was $1.78 \mathrm{~Gy}(95 \%$ confidence intervals $(\mathrm{Cl}): 1.59-1.98, P<0.001)$. There were 6,822 patients in the moderately hypofractionated radiotherapy group. The $\alpha / \beta$ ratio was $3.46 \mathrm{~Gy}(95 \% \mathrm{Cl}: 3.08-3.83, P<0.001)$. In the SBRT group of 1,827 patients, the $\alpha / \beta$ ratio was $4.24 \mathrm{~Gy}(95 \% \mathrm{Cl}: 4.10-4.39, P<0.001)$. Hence, the calculated $\alpha / \beta$ ratios for $P C a$ tended to become higher when the dose per fraction increased. However, the $\mathrm{k}$ and a values were not affected by fractionation schedules. The $\mathrm{k}$ value was calculated as 5.35 (95\% Cl: 4.61-6.08, $P<0.001), 1.15$ (95\% Cl: 0.21-2.09, $P=0.017$ ), and 1.67 (95\% Cl: $-4.80-8.15, P<0.61)$, respectively, in patients receiving conventionally fractionated radiotherapy, moderately hypofractionated radiotherapy and SBRT. The a value was $0.043 \mathrm{~Gy}^{-1}$ (95\% Cl: $\left.0.029-0.056, P<0.001\right), 0.026 \mathrm{~Gy}^{-1}$ (95\% Cl: $\left.0.016-0.036, P<0.001\right)$, and $0.042 \mathrm{~Gy}^{-1}\left(95 \% \mathrm{Cl}^{-}-0.27-\right.$ $0.36, P<0.79)$, respectively.

Table 5

Parameters estimated with $95 \%$ Cls in different regimens of radiotherapy

\begin{tabular}{|c|c|c|c|c|c|c|c|c|c|}
\hline & \multicolumn{3}{|l|}{ k } & \multicolumn{3}{|l|}{$a\left(G y^{-1}\right)$} & \multicolumn{3}{|l|}{$\alpha / \beta$ (Gy) } \\
\hline & Estimate & $95 \% \mathrm{Cl}$ & $\mathbf{P}$ & Estimate & $95 \% \mathrm{Cl}$ & $\mathbf{P}$ & Estimate & $95 \% \mathrm{Cl}$ & $P$ \\
\hline $\begin{array}{l}\text { Conventional } \\
\text { fractionation }\end{array}$ & 5.35 & $\begin{array}{l}4.61- \\
6.08\end{array}$ & $<001$ & 0.043 & $\begin{array}{l}0.029- \\
0.056\end{array}$ & $<.001$ & 1.78 & $\begin{array}{l}1.59- \\
1.98\end{array}$ & $\begin{array}{l}<.001 \\
0.001\end{array}$ \\
\hline $\begin{array}{l}\text { Moderate } \\
\text { hypofractionation }\end{array}$ & 1.15 & $\begin{array}{l}0.21- \\
2.09\end{array}$ & 0.017 & 0.026 & $\begin{array}{l}0.016- \\
0.036\end{array}$ & $<001$ & 3.46 & $\begin{array}{l}3.08- \\
3.83\end{array}$ & $\begin{array}{l}< \\
0.001\end{array}$ \\
\hline SBRT & 1.67 & $\begin{array}{l}-4.80- \\
8.15\end{array}$ & 0.61 & 0.042 & $-0.27-0.36$ & 0.79 & 4.24 & $\begin{array}{l}4.10- \\
4.39\end{array}$ & $\begin{array}{l}< \\
0.001\end{array}$ \\
\hline
\end{tabular}

Only 21 of 45 studies (14,641 PCa patients) could be grouped by the risks of PCa. For different risk subgroups, the results were shown in Table 6. At the same fractionation schedules, there were no regular changes or significant differences in $\alpha / \beta$ values among the three risks groups. For example, the $\alpha / \beta$ ratios were $1.66 \mathrm{~Gy}(1.48-1.83, P<0.001), 2.29 \mathrm{~Gy}(2.12-2.47, P<0.001)$, and $0.95 \mathrm{~Gy}(0.92-0.99, P$ $<0.001$ ) in the three risk groups, respectively, in the conventionally fractionated radiotherapy group. The calculated k value was 10.2 (95\% Cl:7.34-13.1, $P<0.001$ ), 8.20 (95\% Cl: 6.85-9.56, $P<0.001$ ), and 4.31 (95\% Cl: 2.80-5.83, $P<0.001$ ), respectively, in the low-, intermediate-, and high-risk groups in the conventionally fractionated radiotherapy group and the a value was $0.081 \mathrm{~Gy}^{-1}(95 \% \mathrm{Cl}$ : 0.012-0.15, $P=0.022), 0.073 \mathrm{~Gy}^{-1}$ (95\% Cl: $\left.0.041-0.10, P<0.001\right)$ and $0.023 \mathrm{~Gy}^{-1}(95 \% \mathrm{Cl}:-0.0053-0.051, P=0.11)$, respectively. 
According to the results, we found that the $k$ and a values tended to decrease when the risks of PCa increased. In the moderately hypofractionated radiotherapy group, the same conclusion could be drawn. In the SBRT groups, the $\alpha / \beta$ ratios were $-10.7 \mathrm{~Gy}(95 \% \mathrm{Cl}$ : -12.6-8.7, $P<0.001), 25.6 \mathrm{~Gy}(95 \% \mathrm{Cl}: 21.6-29.6, P<0.001)$, and $2.94 \mathrm{~Gy}(95 \% \mathrm{Cl}:-14.4-20.2, P=0.74)$ in the low-, intermediate-, and high-risk groups, respectively. Since the $\alpha / \beta$ ratio in the low-risk patients was negative, we imposed non-negativity restrictions; thereafter, the $\alpha / \beta$ ratio in the low-risk group was $0.032 \mathrm{~Gy}(95 \% \mathrm{Cl}:-0.40-0.47, P=0.89)$. The conclusion which came out from the conventionally fractionated radiotherapy group and moderately hypofractionated radiotherapy group could not be drawn in the SBRT group due to the limited number of articles involved.

Table 6

Parameters estimated with $95 \% \mathrm{Cls}$ in different risks under different regimens of radiotherapy

\begin{tabular}{|c|c|c|c|c|c|c|c|c|c|c|}
\hline \multirow{2}{*}{$\begin{array}{l}\text { Fractionation } \\
\text { regimen }\end{array}$} & \multirow[t]{2}{*}{ Risk group } & \multicolumn{3}{|l|}{ k } & \multicolumn{3}{|l|}{$a\left(G y^{-1}\right)$} & \multicolumn{3}{|l|}{$\alpha / \beta$ (Gy) } \\
\hline & & Estimate & $\begin{array}{l}95 \% \\
\mathrm{Cl}\end{array}$ & $\mathrm{P}$ & Estimate & $95 \% \mathrm{Cl}$ & $\mathrm{P}$ & Estimate & $\begin{array}{l}95 \% \\
\mathrm{Cl}\end{array}$ & $P$ \\
\hline \multirow[t]{3}{*}{$\begin{array}{l}\text { Conventional } \\
\text { fractionation }\end{array}$} & Low risk & 10.2 & $\begin{array}{l}7.34- \\
13.1\end{array}$ & $<.001$ & 0.081 & $\begin{array}{l}0.012- \\
0.15\end{array}$ & 0.022 & 1.66 & $\begin{array}{l}1.48- \\
1.83\end{array}$ & <. \\
\hline & $\begin{array}{l}\text { Intermediate } \\
\text { risk }\end{array}$ & 8.20 & $\begin{array}{l}6.85- \\
9.56\end{array}$ & $<.001$ & 0.073 & $\begin{array}{l}0.041- \\
0.10\end{array}$ & $<.001$ & 2.29 & $\begin{array}{l}2.12- \\
2.47\end{array}$ & $<.001$ \\
\hline & High risk & 4.31 & $\begin{array}{l}2.80- \\
5.83\end{array}$ & $<.001$ & 0.023 & $\begin{array}{l}-0.0053- \\
0.051\end{array}$ & 0.11 & 0.95 & $\begin{array}{l}0.92- \\
0.99\end{array}$ & <. 001 \\
\hline \multirow[t]{3}{*}{$\begin{array}{l}\text { Moderate } \\
\text { hypofractionation }\end{array}$} & Low risk & 7.68 & $\begin{array}{l}6.15- \\
9.22\end{array}$ & $<.001$ & 0.047 & $\begin{array}{l}0.026- \\
0.067\end{array}$ & $<.001$ & 1.10 & $\begin{array}{l}1.04- \\
1.15\end{array}$ & $<.001$ \\
\hline & $\begin{array}{l}\text { Intermediate } \\
\text { risk }\end{array}$ & 6.62 & $\begin{array}{l}5.85- \\
7.38\end{array}$ & $<.001$ & 0.044 & $\begin{array}{l}0.032- \\
0.057\end{array}$ & $<.001$ & 1.69 & $\begin{array}{l}1.01- \\
2.38\end{array}$ & $<.001$ \\
\hline & High risk & 4.93 & $\begin{array}{l}4.00- \\
5.87\end{array}$ & $\begin{array}{l}<.001 \\
0.00\end{array}$ & 0.011 & $\begin{array}{l}0.0002- \\
0.022\end{array}$ & 0.046 & 0.39 & $\begin{array}{l}0.33- \\
0.45\end{array}$ & <. \\
\hline \multirow[t]{3}{*}{ SBRT } & Low risk & -4.81 & $\begin{array}{l}-16.3- \\
6.64\end{array}$ & 0.41 & -0.14 & $\begin{array}{l}-0.66- \\
0.39\end{array}$ & 0.61 & -10.7 & $\begin{array}{l}-12.6- \\
-8.7\end{array}$ & $<.001$ \\
\hline & $\begin{array}{l}\text { Intermediate } \\
\text { Risk }\end{array}$ & 10.7 & $\begin{array}{l}3.16- \\
18.2\end{array}$ & 0.005 & 0.27 & $\begin{array}{l}-0.019- \\
0.56\end{array}$ & 0.067 & 25.6 & $\begin{array}{l}21.6- \\
29.6\end{array}$ & <. \\
\hline & High risk & 16.0 & $\begin{array}{l}-42.7- \\
74.8\end{array}$ & 0.59 & 0.14 & $\begin{array}{l}-0.89- \\
1.17\end{array}$ & 0.79 & 2.94 & $\begin{array}{l}-14.4- \\
20.2\end{array}$ & 0.74 \\
\hline
\end{tabular}

The preliminary results of verification of fitting results were shown in Table 7. The $X^{2}$ were all $<1$ in all three risk groups and the $P$ values were all $>0.995$ that meant there was no statistical difference between the calculated TCP and the TCP from the original study. In other words, our fitting was accurate.

Table 7

Preliminary results of verification of fitting results

\begin{tabular}{|llllll|}
\hline Fractionation regimen & $\mathbf{k}$ & $\mathbf{a}\left(G^{-1}\right)$ & $\mathbf{a} / \boldsymbol{\beta}$ ratio $(G \mathbf{y})$ & $\mathbf{X}^{2}$ & $\boldsymbol{P}$ value (goodness of fit test) \\
\hline Conventional fractionation & 5.35 & 0.043 & 1.78 & 0.10 & $>0.995$ \\
\hline Moderate hypofractionation & 1.15 & 0.026 & 3.46 & 0.51 & $>0.995$ \\
\hline SBRT & 1.67 & 0.042 & 4.24 & 0.01 & $>0.995$ \\
\hline
\end{tabular}

In summary, for PCa patients receiving conventionally fractionated radiotherapy, moderately hypofractionated radiotherapy, and SBRT, the mean $\alpha / \beta$ ratios were $1.78,3.46$, and $4.24 \mathrm{~Gy}$, respectively. Meanwhile, as the risks of PCa increased, the $k$ and a values decreased.

\section{Discussion}


The $\alpha / \beta$ ratio proposed in the early 1970 's derives from the LQ models $[27,28]$. Factors that can influence $\alpha$ and/or $\beta$ independently increase or decrease the $\alpha / \beta$ ratio. The major influencing factors are internal factors from cells themselves and external factors from physical or chemical effects $[17,29]$. The internal factors include cell cycle regulation, cell repopulation, and DNA damage repair after irradiation. The external physical factors include temperature (hyperthermia), oxygenation (hypoxia), characteristics of radioactive rayslike linear energy transfer, and the dose rate. The external chemical factors are some anticancer drugs such as cisplatin, EGFR inhibitors, and PARP1 inhibitors. Thus, there are multiple factors that affect the $\alpha / \beta$ ratio and modify the radiosensitivity of tumors.

Our study showed that the $\alpha / \beta$ ratio tended to become higher when the dose per fraction increased. The $\alpha / \beta$ ratios may increase also dynamically during treatment, from approximately $4 \mathrm{~Gy}$ for 'short' fractionation schedules to about $1.5 \mathrm{~Gy}$ for long schedules, which probably reflects the process of accelerated repopulation in normal acute skin reactions [30,31]. For late-responding tissues and slowgrowing tumors like PCa, however, there may be no repopulation during radiotherapy [30], and the $\alpha / \beta$ ratio increase is not due to tumor cells repopulation. Also the time factors should not be considered in late-responding tissues [32,33]. Thus, we did not take the time factor into consideration when converting doses using the standard LQ model.

Recent randomized trials demonstrated that hypofractionated radiotherapy was not superior to conventional radiotherapy in PCa. In the Radiation Therapy Oncology Group (RTOG) 0415 [34], Hypofractionated Irradiation for Prostate Cancer trial (HYPRO) [24], and the Fox Chase trial (ClinicalTrials.gov identifier: NCT00062309) [35], biological effective doses (BEDs) in hypofractionated vs. conventionally fractionated radiotherapy groups were calculated as 186.7 vs. $162.4 \mathrm{~Gy}, 211.0$ vs $182.0 \mathrm{~Gy}$, and 196.6 vs $177.3 \mathrm{~Gy}$, respectively, using an $\alpha / \beta$ ratio of $1.5 \mathrm{~Gy}$. All BEDs in the hypofractionated groups were 19-29 Gy higher than BEDs of the conventionally fractionated groups. Nevertheless, the higher BEDs did not lead to satisfactory improvements in the outcome. This may be attributable to the inaccurate conversion of radiation doses using the LQ model. In our study, the $\alpha / \beta$ ratio tended to become higher when the dose per fraction increased. When the doses in the three trials were converted with the LQ model using the $\alpha / \beta$ ratios that we estimated (1.78 Gy for conventionally fractionation and $3.46 \mathrm{~Gy}$ for moderate hypofractionation), BEDs of the hypofractionated and conventionally fractionated groups were 120.6 and $148.4 \mathrm{~Gy}, 128.1$ and $165.6 \mathrm{~Gy}$, and 125 and $161.4 \mathrm{~Gy}$ in the RTOG0415, HYPRO, and Fox Chase trial, respectively. The BEDs in the hypofractionated group were significantly lower than in the conventionally fractionated group. Thus, the non-superiority of the hypofractionated group could be in part explained by these BEDs calculated based on our results.

Several studies investigated the appropriateness of the LQ model at high doses per fraction. Previous in vitro and in vivo studies demonstrated that the LQ model overestimated the efficacy of tumor cell killing with a high dose per fraction $[3,36,37]$. Thus, several models were proposed modifying the standard LQ model to reasonably convert conventionally fractionated doses to equivalent single or hypofractionated doses. The lethal-potentially-lethal (LPL) model considered DNA lesion repair and could explain very effectively the shoulder on survival curves [38]; The modified LQ (MLQ) model made a better fit to the iso-effect data than the LQ model in a single high dose [39]. The "universal survival curve"(USC) model proposed by Park et al. combined two classical radiobiological models: the multitarget model and the standard LQ model that provide superior approximation of survival curves in the high-dose range. [40], and generalized LQ (gLQ) model encompasses the full dose range of possible dose delivery patterns and special radiotherapy schemes. [3]. Characteristics of these models have already been described [5]. Wang et al. [3] demonstrated that the problems in the LQ model derived from the amount of sublethal damage were reduced owing to conversion to lethal damage at a single high dose; if sublethal damage is converted to lethal damage, then the $\alpha / \beta$ ratio is elevated with a single high dose according to the definition of the $\alpha / \beta$ ratio. Other studies also revealed that cell death at high doses exceeded the probability of intracellular cell repair, and higher $\alpha / \beta$ ratios were shown with a linear survival curve [38, 41]. An in vivo study involving a murine tumor model demonstrated that an equivalent single high dose converted from fractionated radiotherapy was lower than the actual dose. However, when a higher $\alpha / \beta$ ratio was used, the discrepancy became smaller[37]. Our data agreed with their results. At different fractional doses, the $\alpha / \beta$ ratio tended to be higher when the dose per fraction increased (1.78 Gy for conventional fractionation, 3.46 Gy for moderate hypofractionation, and 4.24 Gy for SBRT). Especially in the SBRT groups, the high $\alpha / \beta$ ratio was marked.

We also found that $\mathrm{a}$ and $\mathrm{k}$ values decreased with risk elevation in the conventional fractionation and moderate hypofractionation groups. These results were similar to those in the previous study [15]. A decrease in the a values with escalation of the risk group can be attributed to higher radio-resistance of tumor cells in higher risk patients. $k$ represents the natural logarithm of an effective target cell number, and a decrease in $\mathrm{k}$ values means that the effective target cell number is reduced with escalation of the risk group.

The $\alpha / \beta$ ratio in the low-risk patients of the SBRT group was in the negative range. A study using external beam radiation therapy alone also had negative $\alpha / \beta$ ratios [42]. Repeated measures of PSA at 6 institutions were analyzed and data from 3 institutions including RTOG showed negative $\alpha / \beta$ ratios. In the Peter MacCallum Cancer Center, the $\alpha / \beta$ ratio was $-2.05 \mathrm{~Gy}(95 \% \mathrm{Cl}:-\infty-\infty)$. Another study

Page 11/15 
found that the $\alpha / \beta$ ratio of arteriovenous malformation obliteration after radiosurgery was markedly negative $(\alpha / \beta=-49.3 \pm 5.3)[43]$. However, neither study explained why the $\alpha / \beta$ ratio was negative. These results as well as ours suggest a limitation of this calculation method in that it could possibly yield unrealistic $\alpha / \beta$ ratios, especially when the patient number is small.

There are several limitations in our study. Since we divided the whole group into three subgroups according to the fractionation schedule, the dose ranges per fraction were relatively narrow in each fractionation group. This may increase the variability of the estimated $\alpha / \beta$ ratios, but we tried to solve this problem by including as many patients as possible. Subtle variations in patient evaluation including the definition of PSA failure and treatment including the dose prescription method among respective studies would also contribute to variability in the estimated $\alpha / \beta$ ratios; this problem is common to all studies of this kind, and is considered to be ameliorated by including a large number of patients. An analysis of over 14,000 patients showed that the derived $\alpha / \beta$ ratios were not different between studies using the ASTRO definition and those using the Phoenix definition [44]. The patient number in our study was larger than in any other studies investigating the $\alpha / \beta$ ratio for PCa. Also, the influence of ADT was not considered, as was the case with other previous studies, since a previous study indicated the minimal influence of ADT [15].

In conclusion, our study using mathematical statistics with 5y-bRFS data in PCa patients demonstrated that the $\alpha / \beta$ ratio was dependent on the fractionation schedule. In SBRT, the estimated $\alpha / \beta$ ratio was $>4 \mathrm{~Gy}$. Therefore, to convert conventionally fractionated radiation doses to an equivalent single high dose, it may be necessary to use either a modified formula or a higher $\alpha / \beta$ ratio with the standard LQ model.

\section{Abbreviations}

\begin{tabular}{|ll|}
\hline Abbreviation & Meaning \\
\hline PCa & Prostate Cancer \\
\hline LQ & Linear-Quadratic \\
\hline TCP & Tumor Control Probability \\
\hline SBRT & Stereotactic Body Radiotherapy \\
\hline IMRT & Intensity-Modulated Radiotherapy \\
\hline ADT & Androgen Deprivation Therapy \\
\hline 5y-bRFS & 5-Year Biochemical Relapse-Free Survival \\
\hline NCCN & National Comprehensive Cancer Network \\
\hline PSA & Prostate Specific Antigen \\
\hline CI & Confidence Intervals \\
\hline RTOG & Radiation Therapy Oncology Group \\
\hline BEDs & Biological Effective Doses \\
\hline HYPRO & Hypofractionated Irradiation for Prostate Cancer Trial \\
\hline LPL & Lethal-Potentially-Lethal \\
\hline MLQ & Modified LQ \\
\hline USC & Universal Survival Curve \\
\hline gLQ & generalized LQ \\
\hline
\end{tabular}

\section{Declarations}

Ethical Approval and Consent to participate: Not applicable.

Consent for publication: All authors have agreements on publication.

Availability of supporting data: Not applicable. 
Funding: This research was funded in part by the Japanese Ministry of Education, Culture, Sports, Science and Technology.

Authors' contributions: Corresponding author: Xian-Shu Gao and Yuta Shibamoto contributed equally to this paper.

Acknowledgements: The authors with to thank Prof. Zhi Geng, Dr. Yunjian Yin, and Dr. Han Wang (School of Mathematical Sciences, Peking University) for their valuable advices on the statistical methods.

\section{References}

1. Gorayski P, Pinkham MB, Lehman M: Advances in radiotherapy technology for prostate cancer: What every GP should know. Australian Family Physician 2015, 44(9):663-667.

2. Joiner MC, Bentzen SM. Fractionation: the linear-quadratic approach. In: Joiner M, van der Kogel A, eds. Basic Clinical Radiobiology. London, Hodder Arnold; 2009:102-119.

3. Wang JZ, Huang Z, Lo SS et al: A generalized linear-quadratic model for radiosurgery, stereotactic body radiation therapy, and highdose rate brachytherapy. Sci Transl Med 2010, 2(39):39ra48.

4. Kirkpatrick JP, Meyer JJ, Marks LB: The linear-quadratic model is inappropriate to model high dose per fraction effects in radiosurgery. Seminars in radiation oncology 2008, 18(4):240-243.

5. Shibamoto Y, Otsuka S, Iwata H et al: Radiobiological Evaluation of the Radiation Dose as Used in High-precision Radiotherapy: Effect of Prolonged Delivery Time and Applicability of the Linear-quadratic Model. Journal of Radiation Research 2012, 53(1):1-9.

6. Miyakawa A, Shibamoto Y, Otsuka $S$ et al: Applicability of the linear-quadratic model to single and fractionated radiotherapy schedules: an experimental study. J Radiat Res 2014, 55(3):451-454.

7. Brenner DJ: The linear-quadratic model is an appropriate methodology for determining isoeffective doses at large doses per fraction. Seminars in radiation oncology 2008, 18(4):234-239.

8. Guckenberger M, Klement RJ, Allgauer $\mathrm{M}$ et al: Applicability of the linear-quadratic formalism for modeling local tumor control probability in high dose per fraction stereotactic body radiotherapy for early stage non-small cell lung cancer. Radiotherapy and oncology : journal of the European Society for Therapeutic Radiology and Oncology 2013, 109(1):13-20.

9. Brenner DJ, Hall EJ: Fractionation and protraction for radiotherapy of prostate carcinoma. Int J Radiat Oncol Biol Phys 1999, 43(5):1095-1101.

10. Fowler J, Chappell R, Ritter M: Is alpha/beta for prostate tumors really low? Int J Radiat Oncol Biol Phys 2001, 50(4):1021-1031.

11. Kal HB, Van Gellekom MPR: How low is the $\alpha / \beta$ ratio for prostate cancer? International Journal of Radiation Oncology*Biology*Physics 2003, 57(4):1116-1121.

12. Vogelius IR, Bentzen SM: Meta-analysis of the alpha/beta ratio for prostate cancer in the presence of an overall time factor: bad news, good news, or no news? Int J Radiat Oncol Biol Phys 2013, 85(1):89-94.

13. Consensus statement: guidelines for PSA following radiation therapy. American Society for Therapeutic Radiology and Oncology Consensus Panel. Int J Radiat Oncol Biol Phys 1997, 37(5):1035-1041.

14. Roach M, 3rd, Hanks G, Thames H, Jr. et al: Defining biochemical failure following radiotherapy with or without hormonal therapy in men with clinically localized prostate cancer: recommendations of the RTOG-ASTRO Phoenix Consensus Conference. Int J Radiat Oncol Biol Phys 2006, 65(4):965-974.

15. Miralbell R, Roberts SA, Zubizarreta E et al: Dose-fractionation sensitivity of prostate cancer deduced from radiotherapy outcomes of 5,969 patients in seven international institutional datasets: alpha/beta $=1.4$ (0.9-2.2) Gy. Int J Radiat Oncol Biol Phys 2012, 82(1):e17-24.

16. Roberts SA, Miralbell R, Zubizarreta EH et al: A modelled comparison of prostate cancer control rates after high-dose-rate brachytherapy (3145 multicentre patients) combined with, or in contrast to, external-beam radiotherapy. Radiotherapy and oncology : journal of the European Society for Therapeutic Radiology and Oncology 2014, 111(1):114-119.

17. Barendsen GW, Van Bree C, Franken NA: Importance of cell proliferative state and potentially lethal damage repair on radiation effectiveness: implications for combined tumor treatments (review). Int J Oncol 2001, 19(2):247-256.

18. Roberts SA, Hendry JH: A realistic closed-form radiobiological model of clinical tumor-control data incorporating intertumor heterogeneity. Int J Radiat Oncol Biol Phys 1998, 41(3):689-699.

Page $13 / 15$ 
19. Eade TN, Hanlon AL, Horwitz EM et al: What dose of external-beam radiation is high enough for prostate cancer? Int J Radiat Oncol Biol Phys 2007, 68(3):682-689.

20. Dearnaley D, Syndikus I, Mossop H et al: Conventional versus hypofractionated high-dose intensity-modulated radiotherapy for prostate cancer: 5-year outcomes of the randomised, non-inferiority, phase 3 CHHiP trial. The Lancet Oncology 2016, 17(8):10471060.

21. Zelefsky MJ, Yamada Y, Fuks Z et al: Long-term results of conformal radiotherapy for prostate cancer: impact of dose escalation on biochemical tumor control and distant metastases-free survival outcomes. Int J Radiat Oncol Biol Phys 2008, 71(4):1028-1033.

22. Catton CN, Lukka H, Gu CS et al: Randomized Trial of a Hypofractionated Radiation Regimen for the Treatment of Localized Prostate Cancer. J Clin Oncol 2017, 35(17):1884-1890.

23. King CR, Freeman D, Kaplan I et al: Stereotactic body radiotherapy for localized prostate cancer: pooled analysis from a multiinstitutional consortium of prospective phase II trials. Radiotherapy and oncology : journal of the European Society for Therapeutic Radiology and Oncology 2013, 109(2):217-221.

24. Incrocci L, Wortel RC, Alemayehu WG et al: Hypofractionated versus conventionally fractionated radiotherapy for patients with localised prostate cancer (HYPRO): final efficacy results from a randomised, multicentre, open-label, phase 3 trial. The Lancet Oncology 2016, 17(8):1061-1069.

25. Mai W, Blanco A, Smiedala M et al: Long-term Outcome of a Moderate-Hypofractionated Intensity Modulated Radiation Therapy (IMRT) Approach Utilizing an Endorectal Balloon for Patients with Localized Prostate Cancer. International Journal of Radiation Oncology*Biology*Physics 2010, 78(3).

26. Kupelian PA, Willoughby TR, Reddy CA et al: Hypofractionated intensity-modulated radiotherapy (70 Gy at 2.5 Gy per fraction) for localized prostate cancer: Cleveland Clinic experience. Int J Radiat Oncol Biol Phys 2007, 68(5):1424-1430.

27. A. M. Kellerer HHR: The theory of dual radiation action. Curr Top Radiat Res 1972:74.

28. Chadwick KH, Leenhouts HP: A molecular theory of cell survival. Phys Med Biol 1973, 18(1):78-87.

29. Franken NAP, Oei AL, Kok HP et al: Cell survival and radiosensitisation: Modulation of the linear and quadratic parameters of the LQ model. International Journal of Oncology 2013, 42(5):1501-1515.

30. Jones B, Dale R: The evolution of practical radiobiological modelling. The British journal of radiology 2019, 92(1093):20180097.

31. Hopewell JW, Nyman J, Turesson I: Time factor for acute tissue reactions following fractionated irradiation: a balance between repopulation and enhanced radiosensitivity. International journal of radiation biology 2003, 79(7):513-524.

32. Fowler JF: The linear-quadratic formula and progress in fractionated radiotherapy. Br J Radiol 1989, 62(740):679-694.

33. Jones L, Hoban P, Metcalfe P: The use of the linear quadratic model in radiotherapy: a review. Australasian physical \& engineering sciences in medicine 2001, 24(3):132-146.

34. Lee WR, Dignam JJ, Amin MB et al: Randomized Phase III Noninferiority Study Comparing Two Radiotherapy Fractionation Schedules in Patients With Low-Risk Prostate Cancer. J Clin Oncol 2016, 34(20):2325-2332.

35. Pollack A, Walker G, Horwitz EM et al: Randomized trial of hypofractionated external-beam radiotherapy for prostate cancer. J Clin Oncol 2013, 31(31):3860-3868.

36. Iwata H, Shibamoto Y, Murata R et al: Estimation of errors associated with use of linear-quadratic formalism for evaluation of biologic equivalence between single and hypofractionated radiation doses: an in vitro study. Int J Radiat Oncol Biol Phys 2009, 75(2):482-488.

37. Otsuka S, Shibamoto Y, Iwata H et al: Compatibility of the linear-quadratic formalism and biologically effective dose concept to high-dose-per-fraction irradiation in a murine tumor. Int J Radiat Oncol Biol Phys 2011, 81(5):1538-1543.

38. Curtis SB: Lethal and Potentially Lethal Lesions Induced by Radiation - a Unified Repair Model. Radiation research 1986, 106(2):252-270.

39. Guerrero M, Li XA: Extending the linear-quadratic model for large fraction doses pertinent to stereotactic radiotherapy. Physics in Medicine and Biology 2004, 49(20):4825-4835.

40. Park C, Papiez L, Zhang S et al: Universal survival curve and single fraction equivalent dose: useful tools in understanding potency of ablative radiotherapy. Int J Radiat Oncol Biol Phys 2008, 70(3):847-852.

41. Fowler JF: Linear quadratics is alive and well: in regard to Park et al. (Int J Radiat Oncol Biol Phys 2008;70:847-852). Int J Radiat Oncol Biol Phys 2008, 72(3):957; author reply 958. 
42. Proust-Lima C, Taylor JM, Secher S et al: Confirmation of a low alpha/beta ratio for prostate cancer treated by external beam radiation therapy alone using a post-treatment repeated-measures model for PSA dynamics. Int J Radiat Oncol Biol Phys 2011, 79(1):195-201.

43. Flickinger JC, Kondziolka D, Maitz AH et al: An analysis of the dose-response for arteriovenous malformation radiosurgery and other factors affecting obliteration. Radiotherapy and oncology : journal of the European Society for Therapeutic Radiology and Oncology 2002, 63(3):347-354.

44. Dasu A, Toma-Dasu I: Prostate alpha/beta revisited - an analysis of clinical results from 14168 patients. Acta Oncol 2012 , 51(8):963-974.

\section{Supplementary Files}

This is a list of supplementary files associated with this preprint. Click to download.

- Supplementarymaterial.docx 